\title{
Daño emergente futuro. Corte Suprema, sentencia de fecha 14 de septiembre de 2017, Rol N400-2017
}

\section{Future emerging damage. Supreme Cour judgment dated September 14, 2017, Roll No 400-2017}

\author{
TOMÁs FrencK ${ }^{1}$
}

\section{RESUMEN}

El presente comentario analiza la sentencia dictada por la Corte Suprema el 14 de septiembre de 2017, en que la Corte, conociendo recurso de casación en el fondo, se refirió sobre la posibilidad de indemnizar un daño emergente que se producirá en el futuro. Esta sentencia resulta peculiar, en tanto que tendemos a pensar el daño emergente como aquella indemnización que deriva de un perjuicio pecuniario ya consumado o efectivo. Sin embargo, en este caso la Corte Suprema estimó que el daño emergente que se producirá en el futuro en tanto exista certeza de su ocurrencia futura, puede ser indemnizado.

Palabras claves: Derecho civil, indemnización de perjuicios, daño emergente.

\section{SUMMARY}

This commentary analyses the Supreme Court's ruling of 14 September 2017, in which the Court, hearing an appeal, referred to the feasibility of compensating direct damages that will occur in the future. This ruling is unique since we tend to think of the direct damage as compensation derives from a pecuniary loss already incurred. However, in this case, the Supreme Court considers that direct damage that will occur in the future, insofar as there is a certainty of it, could be compensated.

Keywords: Private law; compensation; future damages. 


\section{Introducción}

Como advierte el profesor Barros (2006), el daño es "condición indispensable bajo cualquier régimen de responsabilidad civil" (p. 215). No obstante, a pesar de la importancia del daño como requisito ineludible de la responsabilidad civil, son escasas las normas de nuestro código dedicadas a este aspecto. Así, el derecho de dańos es una materia en que la literatura ha realizado grandes esfuerzos interpretativos al momento de dilucidar la naturaleza, especie y monto de los perjuicios que corresponden ser indemnizados al momento de verificarse un incumplimiento contractual o un hecho ilícito.

A modo de ejemplo, la precaria regulación en materia de daños ha llevado necesariamente a la doctrina a establecer más y nuevas categorías de perjuicios. En un primer momento, lo fue con el daño moral en materia civil extracontractual — ahí por la década del 1920 - y hoy con el desarrollo más reciente del concepto de la pérdida de una chance u oportunidad ${ }^{2}$. El tema que analizaremos, en estricto rigor, no dice relación con un nuevo tipo o categoría de daño, sino que más bien se refiere a un alcance adicional que puede adquirir el tradicional dańo emergente. De todas formas, debemos advertir que el concepto de "daño" es unitario en ambos regímenes de responsabilidad civil (Rodríguez, 2012, pp. 215-218), sin embargo, para efectos de este trabajo nos referiremos únicamente al daño en sede contractual.

Como es sabido, el artículo 1556 del Código Civil clasifica a los daños en dos: lucro cesante y daño emergente. El daño emergente —en materia contractual—, según define el profesor Rodríguez Grez (2012), vendría a ser

la diferencia que se produce en el activo del patrimonio de una persona, como consecuencia del incumplimiento contractual, entre el valor del derecho antes y después del incumplimiento que experimenta el patrimonio del acreedor como consecuencia del incumplimiento de una obligación emanada de un contrato e inejecución de la prestación convenida. (p. 216).

El lucro cesante, en cambio, es la "frustración de una legítima utilidad que hubiera incrementado el patrimonio de no haber sucedido el hecho dańoso" (Corral, 2011, p. 148) o, llevándolo a materia contractual, "consiste, entonces, en una proyección en el tiempo de los efectos del incumplimiento" (Rodríguez, 2012, p. 227).

El lucro cesante, tal como se infiere de su definición, siempre va a ser un daño futuro; en cambio, el daño emergente es un daño presente, aun cuando este se produzca en el futuro (Rodríguez, 2012, p. 226). Este último aspecto del daño emergente es el que ocupa pormenorizadamente la Corte Suprema en el fallo que se expondrá. Si bien la doctrina es conteste ante la posibilidad de indemnizar el daño emergente futuro, en cuanto sea cierto, no existen muchos pronunciamientos jurisprudenciales al respecto; allí radica la importancia del caso que estudiaremos.

\section{El caso}

El caso que se analiza tuvo lugar, en primera instancia, bajo el Rol C-30.832-2011, seguido ante el $16^{a}$ Juzgado Civil de Santiago. Luego, en segunda instancia, bajo el Rol No12.260-2015, seguido ante la Corte de Apelaciones de Santiago. En último término, bajo el Rol No400-2017, en donde la Corte Suprema, conociendo recurso de casación, dictó sentencia con fecha 14 de septiembre de 2017.

Barros Bourie prefiere tratar la pérdida de una chance u oportunidad en sede de causalidad (2006, p. 240). 


\subsection{Hechos}

Arturo Ignacio Videla Jara nació el 4 de enero de 2008 y conforme a la normativa vigente se le efectuó el examen de medicina preventiva para detectar si era o no portador de fenilcetonuria, señalándosele en dicho examen que esta era normal. Sin embargo, a los nueve meses de edad los padres se dieron cuenta de que Arturo estaba muy irritable y de que presentaba un desarrollo en general demasiado lento, que no iba en relación con otros niños de su edad. Se consultó al respecto con distintos pediatras del Hospital Clínico de la Universidad Católica, quienes señalaron que era un problema de inmadurez, ya que los exámenes metabólicos, incluyendo el de PKU, eran normales.

A los dos años, cuando el niño ya tenía un retraso mental evidente y su agresividad iba en aumento, la familia consultó a un neurólogo del Hospital San Borja Arriaran. Allí practicaron a Arturo varios exámenes neurológicos y repitieron las muestras metabólicas, concluyendo que Arturo era portador de fenilcetonuria. Una persona fenilcetonúrica puede desarrollarse sin mayores inconvenientes cognitivos y neurológicos si se elimina de la dieta todas las proteínas de origen animal, como carne, huevo, pescado, leche, y se controla la ingesta de fenilalanina en el resto de los alimentos. Sin embargo, como consecuencia de la negligencia del personal en la Clínica Presbiteriana Madre e Hijo - que consistió, primero, en un error en los exámenes de fenilcetonuria y posterior fallo en el diagnóstico; y segundo, en la omisión de informar los resultados a tiempo cuando se dieron cuenta del error-, alimentaron a Arturo con dieta normal, causándole, sin saber, un retraso mental severo e irreversible.

Lo anterior llevó al menor y a su familia a demandar indemnización de perjuicios en forma autónoma a la Clínica Presbiteriana Madre e Hijo, por la suma total de $\$ 454.068 .000$ por concepto de daño patrimonial y moral. Dentro de la avaluación del dańo patrimonial se demandó por dańo emergente los gastos directos que han debido solventar y que deberán seguir pagando en razón de la enfermedad de Arturo (daños futuros), los que ascienden a $\$ 131.508 .000$, que corresponden a especialistas (neurólogos, nutricionistas y médicos) (\$97.848.000), pañales $(\$ 1.800 .000)$, medicamentos $(\$ 6.480 .000)$, colegio especial $(\$ 22.680 .000)$, exámenes médicos $(\$ 2.700 .000)$.

\subsection{Primera instancia}

El 16 Juzgado Civil de Santiago dio por acreditado todos los hechos y desestimó el argumento principal de la parte demandada, que decía relación con que el hecho culpable fue realizado por el laboratorio que realizó el examen, más no por la clínica que cumplió con el estándar de diligencia al realizar el examen en un laboratorio de prestigio. No obstante, en lo que respecta a los perjuicios indemnizables, el tribunal de primera instancia determinó que la demandante no probó el daño emergente, al no acreditarse que existieron desembolsos efectivos por parte de la familia para tratar la patología. Lo anterior llevó a que solamente se diera lugar a la demanda en lo que respecta al dańo moral, por un total de $\$ 80.000 .000$.

\subsection{Corte de Apelaciones}

La parte demandante interpuso un recurso de apelación en contra de la sentencia definitiva. En lo que respecta al presente comentario de jurisprudencia, la apelación se refirió al rechazo del daño por parte del tribunal a la indemnización por dańo emergente.

Los apelantes señalaron, en un acápite de su presentación, que el tribunal de primera instancia debió haber considerado el daño emergente y futuro derivado de la negligencia de la clínica en el erróneo diagnóstico de Arturo. Argumentaron que al momento de impetrar la demanda no contaban con los suficientes antecedentes para avaluar exactamente los perjuicios futuros que sufriría la familia por el 
tratamiento especial al que debía someterse el menor, producto de su condición. No obstante, señalaron en el recurso que dicha apreciación era posible hacerla en el momento con los documentos que se acompañaron en segunda instancia. Sostuvieron que el menor debería asistir a un centro especial hasta que cumpliese la edad de veinticuatro años; el cálculo entonces debía ser: $\$ 5.887 .200$ (costo anual del centro de rehabilitación), más $\$ 490.600$ (costo de matrícula anual), multiplicado por 21 (Arturo tenía tres ańos cuando se presentó la demanda), lo que haría un total de \$123.631.200 que el sentenciador de primera instancia no tuvo en cuenta.

La Ilma. Corte de Apelaciones dio lugar íntegramente al recurso de apelación interpuesto, en lo que respecta al daño emergente, condenado a la clínica al pago de $\$ 123.631 .200$, que corresponde al pago que deberá incurrir la familia en el centro de rehabilitación para Arturo hasta que este cumpla veinticuatro años.

\subsection{El fallo de la Corte Suprema}

Por último, queda analizar lo resuelto por la Corte Suprema al conocer recurso de casación en el fondo interpuesto por la clínica. No obstante, se debe primero revisar algunos argumentos vertidos por la clínica en el recurso.

a) Recurso de casación en el fondo interpuesto por la clínica

La clínica interpuso recurso de casación en el fondo por infracción de ley, argumentando que la Ilma. Corte de Apelación habría infringido el artículo 1556 del Código Civil. Sostuvieron los recurrentes que se habría aplicado este artículo a una situación no prevista por la norma, lo que produjo un error en el cálculo por la indemnización de daño emergente, haciéndolo extensivo a una situación en donde no hubo un detrimento patrimonial efectivo o, si lo hubo, fue menor y diferente al establecido en la sentencia.

En primer lugar, en su argumentación señalaron que la Ilma. Corte de Apelaciones erró en cálculo de los perjuicios al contabilizar veintiún ańos de tratamiento para Arturo. Se incurre en este yerro, puesto que a la fecha de dictación de la sentencia el menor tenía ocho años y en el período de tres a ocho años en que se desarrolló el juicio fue la propia demandante quien reconoció que no incurrieron en gastos médicos ante su precaria situación económica. Es por ello que, al no haber existido desembolsos efectivos durante esos cinco años, ese período de tiempo no puede ser comprendido para calcular el daño emergente.

En segundo lugar, aseveraron que la sentencia de segunda instancia incurrió en un segundo error al considerar el daño emergente hacia el futuro, puesto que el daño emergente alegado vendría a ser un daño cierto, pero eventual.

El daño emergente — según la recurrente- procede solamente cuando se produzcan desembolsos efectivos que impliquen una disminución patrimonial. La Ilma. Corte de Apelaciones estaría determinando un monto indemnizatorio debido a la proyección de la expectativa de vida de los enfermos de fenilcetonuria, lo que implica suponer como hecho cierto uno de carácter hipotético, proyectando una disminución patrimonial que solo es eventual.

Reafirmaron su argumento en que se utilizó como base de cálculo un centro médico en el cual Arturo no estaba siendo tratado a la época de la dictación de la sentencia, no siendo posible tampoco asegurar la estabilidad de esos montos y valores en el futuro, siendo también la base de cálculo meramente hipotética.

b) Sentencia de casación en el fondo y sentencia de reemplazo 
La Corte Suprema en la sentencia de casación, antes de resolver el recurso, comenzó explicando las distintas clasificaciones del daño, partiendo de la distinción más básica entre daño material y daño moral. En palabras de la Corte Suprema, el daño material se divide entre el lucro cesante y el dańo emergente, cuyos conceptos no son definidos por ley. No obstante, señala el máximo tribunal que, dentro de la órbita de la responsabilidad contractual, "la doctrina ha estimado que el primero consiste en el empobrecimiento real y efectivo que sufre el patrimonio del deudor [debió decir "acreedor"] y, el segundo, lo constituye la utilidad que deja de percibir el acreedor por el incumplimiento o cumplimiento tardío de la obligación".

Después, el excelentísimo tribunal sostuvo que el artículo 2329 del Código Civil, al indicar que por regla general todo daño debe ser indemnizado, estableció un principio general, admitido en todos los sistemas: la reparación ha de ser integral. En efecto, comentó la Corte Suprema, según el principio de restitutio in integrum, "la indemnización ha de comprender la suma que coloque a la parte que ha sido dańada en la misma posición en que estaría si no hubiese sufrido el hecho dañoso por el cual ahora está obteniendo compensación”.

Sin embargo, continuó el sentenciador, este principio en el caso de la responsabilidad contractual sufre una grave limitación en el artículo1558 del Código Civil. La norma citada establece que, salvo la hipótesis de dolo o culpa grave, la víctima solo obtendrá la reparación del dańo directo previsto. El perjuicio directo, en materia contractual, vendría a ser la "consecuencia lógica e inmediata del incumplimiento del deudor".

Por último, la Corte Suprema estableció que los requisitos que debe presentar el daño para ser reparable son: que sea cierto y no meramente eventual, que lesione un derecho subjetivo o un interés legitimado por el ordenamiento jurídico, que sea causado por obra de un tercero, distinto a la víctima, y que no se encuentre reparado.

Así las cosas, después de esta explicación previa, la Corte Suprema resolvió la contienda, señalando:

1. En cuanto al primer argumento de la recurrente, que decía relación con el error en el cálculo para cuantificar lo que se debía pagar por la rehabilitación de Arturo, la Corte Suprema concedió el recurso en esta parte, señalando que la Corte de Apelaciones se equivocó "al estimar como daño emergente atenciones que el menor ya no recibió, determinando erróneamente el monto de una indemnización durante un período en que, si bien existió un detrimento patrimonial, no amerita ser resarcido de la manera que declaran”.

2. En cuanto al segundo argumento, respecto de la procedencia del daño emergente futuro, la Corte Suprema decidió rechazar el recurso de casación, resolviendo al respecto:

Habiéndose establecido que el compromiso cognitivo y conductual derivado de su estado es irreversible y que sólo puede ser atenuado con una atención especializada, necesariamente debe concluirse que tanto el daño generado por el incumplimiento como el detrimento patrimonial asociado a su financiamiento constituyen dańos ciertos y no eventuales. En efecto, la sola circunstancia de tratarse de un dańo presente o futuro no le resta certidumbre pues lo que importa es que no exista duda sobre la existencia del dańo, siendo esa certeza el presupuesto indispensable para su resarcimiento.

Los sentenciadores continuaron diciendo que "los gastos asociados a esas prestaciones acarrean un empobrecimiento patrimonial real y efectivo que sufrirán los demandantes. No se trata de una pérdida de ganancia, sino que constituye un daño emergente - actual y futuro- que debe ser resarcido". Y concluyeron, señalando: 
Se advierte que los juzgadores no han infringido el artículo 1556 del Código Civil al condenar a la demandada al pago de las sumas que señalan por concepto de las atenciones que debe recibir el menor Videla Jara hasta que cumpla la edad de 24 años, ya que el costo de tales tratamientos corresponde a un daño emergente cierto cuya existencia es actual y se mantendrá en el tiempo, atendidas las expectativas de vida de pacientes que sufren esa afección.

En suma, se concedió parcialmente el recurso de casación, solo en lo referido al error de cálculo sobre el momento en que se debía comenzar a contar las prestaciones que debía recibir el menor en el centro de rehabilitación. En cuanto al daño emergente futuro, estimaron que en este caso iba a existir para la víctima un daño cierto y futuro derivado del incumplimiento de la clínica, que consiste en el pago de un centro de rehabilitación para el tratamiento de Arturo. La sentencia de reemplazo condenó a pagar a la clínica la suma de $\$ 92.728 .000$.

\section{Comentario}

\subsection{Sobre el dańo emergente futuro}

En primer lugar, se debe partir de la base que el daño emergente futuro es una posibilidad que nuestro ordenamiento reconoce. En efecto, la sentencia de casación de la Corte Suprema hizo un vasto racionamiento para considerar la procedencia de la reparación de este tipo de daño.

Como hemos señalado y tal como lo define la propia Corte Suprema en este caso, el daño emergente es el empobrecimiento real y efectivo que sufre el patrimonio del acreedor por el incumplimiento o cumplimiento tardío de la obligación. Ahora bien, bajo esta definición, el gran obstáculo que presenta el daño emergente futuro es el requisito de que el daño reparable solo puede ser cierto y no eventual.

Como sostiene el profesor Rodríguez Grez (2012), la certidumbre del daño consiste "en un menoscabo que se ha producido o que con certeza se producirá" (p. 219). Agrega que existen tres requisitos para que el daño sea cierto: que se produzca en razón de una hipótesis (causa) necesaria, que conduzca inevitablemente al daño; que la hipótesis (causa) se funde en el desarrollo normal de los acontecimientos, y que la hipótesis fundante del daño, en el caso de la responsabilidad contractual, considere el incumplimiento como el primer eslabón de la cadena causal que lo provoca (ibid). En materia contractual, la causa evidentemente siempre va a ser el incumplimiento.

Con respecto a la exigencia de certidumbre en el daño futuro, el criterio utilizado por la doctrina es el de la "proyección natural de un estado actual de las cosas" (Barros, 2006, p. 237). En suma, el daño actual es el perjuicio ya operado y subsistente en el patrimonio del damnificado al momento del pronunciamiento judicial. En tanto que el futuro es aquel que todavía no se ha producido, pero que ciertamente existirá luego de la sentencia.

Como adelantábamos, el daño emergente puede ser actual o futuro, en cambio, el lucro cesante siempre es a futuro. No obstante, lo cierto es que la diferencia entre uno y otro, a nuestro entender, va a depender de que en el primero necesariamente ese daño que se va a prolongar o materializar en el futuro va a implicar desembolsos patrimoniales para la víctima. En cambio, en el caso del lucro cesante siempre va a implicar una pérdida de ganancia, un aumento patrimonial hipotético que, de no mediar el dańo, se hubiese producido. 
Como última precisión, debemos señalar que don Enrique Barros (2006) justifica la indemnización de daño futuro por razones de "economía procesal" y evitar una sucesión de juicios en el tiempo (p. 237).

\subsection{El caso resuelto por la Corte Suprema}

Para finalizar daremos nuestra opinión sobre el caso resuelto por la Corte Suprema, comentado hasta aquí. Despejada la duda sobre la admisibilidad del daño emergente futuro como perjuicio susceptible de reparación, debemos estudiar si en este caso era procedente resolver como lo hizo la Corte Suprema.

En primer lugar, a partir del análisis de los hechos, opinamos que la Corte Suprema resolvió correctamente al estimar los supuestos de hecho del caso como uno que podría dar lugar una reparación por daños emergentes futuros. Es cierto que haciendo un juicio normativo bajo el criterio de la "proyección normal o natural de las cosas", es dable concluir que, dado el actual estado de Arturo, padecerá el daño de tener que pagar tratamientos especiales hasta los veinticuatro ańos. Es por ello que en este caso se cumplen los requisitos de la certidumbre y de la no eventualidad del daño: la familia sufrirá un detrimento patrimonial efectivo en el futuro debido a los gastos que deberá incurrir en un tratamiento óptimo para el menor.

A pesar de lo anterior, queremos detenernos en tres puntos sobre los cuales la resolución de la Corte Suprema resulta, al menos, cuestionable. El primero dice relación con que la familia durante el juicio no estaba pagando efectivamente el tratamiento de Arturo en el centro de rehabilitación cuya matrícula y arancel fue utilizada por la Corte Suprema como base de cálculo para cuantificar el daño futuro. En este orden de ideas, resulta controvertible considerar la reparación de ese daño como un "daño emergente futuro", toda vez que, de no mediar la sentencia de la Corte Suprema, no hay antecedentes que acrediten que la familia efectivamente realizará desembolsos patrimoniales efectivos para pagar los tratamientos de Arturo en aquel centro. En consecuencia, los antecedentes del caso no lograron acreditar que un menoscabo patrimonial efectivo se va a producir en el futuro. Esta hipótesis la queremos contrastar con un ejemplo: si fuera el caso que Arturo por su enfermedad hubiese estado pagando por un medicamento durante todo el juicio y este medicamento también se deberá seguir pagando en el futuro, entonces sí se contaría con la certeza de que el daño necesariamente se producirá en el futuro.

En segundo lugar, somos de la opinión que el daño por el cual se solicita la indemnización de perjuicios se circunscribe mejor bajo la hipótesis de una reparación por pérdida de una oportunidad. En efecto, lo que en realidad existió es la pérdida de una chance de no tener que sufrir un perjuicio patrimonial que implica costear el tratamiento de rehabilitación. En este caso, el diagnóstico erróneo implicó un aumento de las probabilidades de riesgo en la ocurrencia del daño (retraso mental) y en definitiva se materializó en los costos de tener que pagar este tratamiento adicional, a lo que la literatura diría que es un caso de pérdida de una oportunidad de no sufrir un perjuicio patrimonial ${ }^{3}$.

La tercera crítica tiene que ver con el concepto de previsibilidad del daño. La Corte Suprema resolvió, sin detenerse en demasía, que en este caso el daño es directo y previsible. En otras palabras, que al momento de contratar (la familia con la clínica) era posible prever que un error médico o, en este caso, el erróneo diagnóstico en el examen de fenilcetonuria culminaría ocasionando los daños alegados. Pues bien, los perjuicios previstos son aquellos que "se previeron o pudieron preverse al tiempo del contrato" (Abeliuk, 2014, p. 1033). Tal como sostiene la doctrina el tema de la previsibilidad, es una cuestión de hecho que

Enrique Barros (2006), ejemplifica precisamente con el diagnóstico médico equivocado, al momento de estudiar la institución de la pérdida de una oportunidad (p. 239). 
queda entregada al criterio del juez. Este debe apreciar si el daño producido era o no posible de prever por la clínica.

Al respecto, consideramos que es discutible que existe por parte de la demandada una previsibilidad en el daño causado o, al menos, que requería de un mayor razonamiento por parte del sentenciador en este aspecto. En efecto, lo que debe ser previsible en los términos del artículo 1558 del Código Civil son los perjuicios. El análisis de previsibilidad entre acción y resultado es apropósito del análisis de la evaluación de la relación causal. Creemos que no era previsible para la clínica que en caso de otorgar un mal diagnóstico a Arturo iba a tener que incurrir en costos de rehabilitación hasta los veinticuatro años. Es por ello que, a nuestro juicio, para la clínica no era posible prever los perjuicios indemnizables. A nuestro entender, este es un daño imprevisible.

En suma, si bien a grandes rasgos el razonamiento del tribunal nos parece correcto al considerar al daño emergente futuro como uno de los perjuicios susceptibles a ser indemnizados, lo cierto es que, al analizar los hechos en concreto, es discutible si en este caso correspondía indemnizar por daño emergente futuro por las razones ya expuestas.

\section{Bibliografía citada}

Abeliuk Manasevich, René (2014): Las obligaciones (Santiago, X)

Barros Bourie, Enrique (2006): Tratado de responsabilidad extracontractual (Santiago, X).

Corral Talciani, Hernán (2011): Lecciones de responsabilidad civil extracontractual (Santiago, X).

Rodríguez Grez, Pablo (2012): Responsabilidad contractual (Santiago, X) 